\section{Case Reports in Ophthalmology}

Case Rep Ophthalmol 2020;11:234-241

DOI: $10.1159 / 000508606$

Published online: June 25, 2020 (c) 2020 The Author(s)

Published by S. Karger AG, Basel www.karger.com/cop

\title{
Development of an Infrared Monitor-Guided Bleb Revision Procedure
}

\author{
Kenji Matsushita Rumi Kawashima Kohji Nishida
}

Department of Ophthalmology, Osaka University Graduate School of Medicine,

\section{Keywords}

Trabeculectomy · Bleb revision · Bleb knife $\cdot$ Infrared light · 5-fluorouracil

\section{Abstract}

The needle revision procedure to address failing filtering blebs is a blind technique that might easily damage the scleral flap, conjunctiva, and choroid. We propose a new surgical procedure, infrared monitor-guided bleb revision, to perform bleb revision minimally invasively and effectively, and demonstrate the procedure in a patient. We developed the guided procedure with the infrared monitor to observe the bleb interior with greater contrast. Under the monitor, we dissect the hard fibrotic tissue with a bleb knife and, if necessary, remove adhesions using a needle and vitreous forceps. Finally, 5 -fluorouracil is injected into the subconjunctiva. We have performed bleb revisions safely with clear visualization of the scleral flap using an infrared light. In the current case, the patient had good intraocular pressure control for about 1 year. The new infrared monitor-guided bleb revision procedure facilitates successful bleb revisions without damage to the underlying structures.

\section{Introduction}

Trabeculectomy is the most popular of the filtering surgeries [1]; however, filtration failure can occur often over time, and the reduced filtering effect results in increased intraocular 


\section{Case Reports in Ophthalmology}

pressure (IOP) in patients with glaucoma [1]. The conjunctival flap in filtering surgery has been modified from a limbal-based to a fornix-based site to avoid the sight-threatening complications of filtration surgery, such as infection [2] and latent hypotony [3]. The internal bleb structure has also changed [3]. Because the limbal-based filter results in an ischemic bleb, the edges of the scleral flap are visible through the thin conjunctiva [3]. However, because the fornix-based bleb often is covered with thick conjunctival epithelium and Tenon tissue, especially if associated with the Tenon advancement method, the internal structure often is not visible [3]. Thus, the needle revision might be a blind technique that can easily damage the scleral flap, conjunctiva, and choroid. In the early postoperative period, needle revision of the filtering bleb is less invasive. However, the scleral flaps in Tenon's cysts and late-stage blebs are surrounded by tight fibrotic collagen tissue, referred to as the ring of steel [1]. Historically, needle revisions were guided by slit-lamp microscopy, which improved the contrast of the internal bleb images [4]. Other methods use a laser suture lysis lens, such as the Mandelkorn lens (Blumenthal Suturelysis; Volk Optical, Inc., Mentor, OH, USA), which can visualize the bleb by compressing the thick conjunctiva. However, these procedures carry the risk of conjunctival tears because of the reduced working space resulting from the limited surgical view or tissue compression.

We propose a new surgical procedure to resolve these issues in which we use a bleb knife (KAI Instruments Ltd., Hong Kong, PR China) instead of a needle to dissect the hard fibrotic tissue, as developed by Sagara [5]. However, the sharpness of the knife might damage the soft ocular surface tissue during blind bleb revision. To prevent this, we developed the infrared monitor-guided bleb revision (IGBR) technique, which uses an infrared monitor to perform the bleb revision with little damage to the scleral flap. Freeing up both hands during this procedure might facilitate the development of new treatment options for use under the scleral flap; e.g., use of small-gauge needles to cut the edge of the scleral flap and insertion of vitreous forceps through the filtering bleb to grasp the edges of the scleral flap and elevate the flap. The video (Supplemental Digital Content 1) demonstrates the IGBR procedure performed with a bleb knife and vitreous forceps. This procedure ultimately may be categorized as a minimally invasive glaucoma surgery.

\section{New Instrument}

The infrared charged coupled device (CCD) camera (XC-EI50; Sony, Tokyo, Japan) is embedded in a standard surgical microscope (OMS-90; Topcon Co., Tokyo, Japan) connected to the monitor (Fig. 1a). The monitor connected to the infrared CCD camera can visualize the site of the scleral filtering flap (Fig. 1b, c). To examine the effect of the infrared ray (IR) on visualization of the scleral flap, we compared the scores assigned to visualization of the visible flap between the images obtained using visible light and infrared light, using a slit-lamp microscopy (Slit) and meibography (IR monitor) and the swept-source anterior-segment optical coherence tomography ([AS-OCT] SS-1000 CASIA; Tomey Corporation, Inc., Nagoya, Japan) (Fig. 2). We scored the visibility of the scleral flap according to the following definitions: (0) indicates not visible; (1) blurry image; and (2) clearly visible. The score for an image was the sum of the three scores for the three sides of each flap. The highest possible score was 6 . We used the Wilcoxon signed-rank test for statistical analyses ( $n=22$ cases). 


\section{Case Reports in Ophthalmology}

\section{Case Presentation}

A 37-year-old male was diagnosed with herpetic keratouveitis based on AS inflammation in his right eye after having flu-like symptoms. The IOP increased to $40 \mathrm{~mm} \mathrm{Hg}$ because of a large peripheral anterior synechia in the right eye, but the inflammation decreased gradually with medical treatment. Three and a half months thereafter, trabeculectomy with mitomycin $\mathrm{C}$ was performed; the IOP increased to $26 \mathrm{~mm} \mathrm{Hg}$ because of filtration failure. We first performed an IGBR without 5-fluorouracil (5-FU); however, when the IOP increased to $24 \mathrm{~mm} \mathrm{Hg}$ again 1 month later, we performed a second IGBR with 5-FU.

We performed the standard one-step method in which only the conjunctival flap was dissected, but in this case with tough scleral fibrosis, we performed the two-step method. A topical anesthetic ( $4 \%$ xylocaine) and $0.033 \%$ polyvinyl alcohol iodine were applied to the eye, and the patient was covered with a sterile drape. We injected $0.5 \mathrm{~mL}$ of $2 \%$ xylocaine with epinephrine into the conjunctiva $10 \mathrm{~mm}$ from the visible scleral flap through a 30-gauge needle and created one conjunctival incision with a bleb knife in the same space. We extended the Tenon tunnel to the scleral flap edge through the conjunctival incision and again inserted vitrectomy forceps. While elevating the flap edge, we opened the fibrotic clot on the flap edge. A subconjunctival injection of 5-FU $(0.1 \mathrm{~mL}, 250 \mathrm{mg} / 5 \mathrm{~mL})$ was administered into the superior bulbar space. Postoperatively, oral antibiotics were prescribed for 4 days, and a topical steroid ( $0.1 \%$ betamethasone sodium phosphate) and an antibiotic ( $1.5 \%$ levofloxacin hydrate) were instilled three times daily. The patient was observed routinely, and the topical steroid was tapered. In this case, the IOP remained below $21 \mathrm{~mm} \mathrm{Hg}$ for 8 months with anti-glaucoma eye drops.

\section{Discussion and Conclusion}

Bleb revision can improve bleb function in cases of bleb failure. Bleb revision with a smallgauge needle originated from conjunctival dialysis reported by Ferrer [6], now referred to as needling, which is an easier procedure. While most studies have reported outcomes of needling performed early in the postoperative period to resurrect failing blebs [7], needling to treat late-onset bleb failure is challenging because of the tough fibrotic tissue around the bleb, and needles larger than 23 gauge are necessary to handle mature bleb failure. Sagara [5] developed a bleb knife for dissections (KAI Instruments Ltd.), the use of which is advantageous to perform treatments of the tough fibrotic tissue on the scleral flap, which is difficult using the previous method. However, the soft ocular tissue can be damaged by the knife when performing blind bleb revision. Thus, the small-incision bleb revision using a bleb knife requires proper guidance.

An infrared light source has been adapted to many types of objective machines such as OCT. In clinic, infrared imaging can visualize the inside of the bleb when the inside is not visible. We observed the internal flap with AS-OCT. Some studies have reported the advantages of three-dimensional OCT-guided bleb revision [8]. However, the technique was limited in that it only could be performed preoperatively. However, a mobile pen-shaped infrared meibography device (Meibom-pen; JFC Sales Plan Co. Ltd., Tokyo, Japan) [9] is now popular for observing the meibomian glands through the palpebral conjunctiva and for inside the scleral flap edge in many cases for which other microscopes with a light source could not be used (Fig. 2a, b). In a visualization test of three lines of the scleral flap reported by Nomura et al. [10], 84.2\% of the cases were visualized using IR images. We observed lines in 95.5 and $100 \%$ in the same 


\section{Case Reports in Ophthalmology}

group of patients, respectively, using meibography (IR monitor) and swept-source AS-OCT, in which 63.6\% were visualized using slit-lamp microscopy (Fig. 2d). The infrared light facilitated visualization of the scleral flaps better than visible light (Fig. 2e). Thus, we developed the IGBR procedure under monitoring using a micro-CCD camera for meibography imbedded in the surgical microscope. During bleb revision, we visualized the scleral flap under infrared light despite strong scarring and eliminated the tight adherence under the scleral flap with the bleb knife and vitreous forceps, and the bleb revision was completed (online suppl. Digital Content 1; for all online suppl. material, see www.karger.com/doi/10.1159/000508606). The main drawback of needling is bleeding as a potential complication during bleb revision. Our technique fared better regarding visualization if and when bleeding occurs. No complications developed during the procedure. After revision, the filtering blebs enlarged and became more diffuse and higher (Fig. 3a-d). AS-OCT showed that the scleral flaps in the revised bleb were undamaged (Fig. 3c, d).

Our needle bleb revision is effective even for use with well-established bleb many years after filtering surgery. The morphology of a late bleb is thick scarring around the scleral flap that sometimes extends even under the flap and can require additional intervention after needling. If so, this flap is elevated, and the path is opened with a needle or forceps such as vitreous forceps. This flap elevation has already been reported, but when we perform this procedure, the infrared light is very effective because it enables adequate identification of the edge of the flap.

The IOP of this patient was $24 \mathrm{~mm}$ Hg before bleb revision with 5-FU and at that time, he had damage in the visual field that was classified as stage 2 of Grave's Modified Method of the Aulhorn Classification. We defined complete success of filtering surgery, including this needle revision, as maintenance of the IOP under the target pressure without additional treatment such as eye drops; we defined qualified success as maintenance of the IOP under the target pressure with medical treatment but without any additional surgery, or sight loss. The target pressure for this patient within the normal level was $21 \mathrm{~mm} \mathrm{Hg}$. Eventually, his pressure was maintained at $21 \mathrm{~mm} \mathrm{Hg}$ until the final visit 8 months after revision. During observation, the visual field was maintained. We believe this target pressure was adequate for maintaining the visual function of this patient. The patient needed an additional bleb revision about 1 year after this revision; his IOP has remained stable at about $15 \mathrm{~mm} \mathrm{Hg}$ for 5 years.

Our system provides a mono-vision view, so it is necessary to confirm the actual procedure with a standard microscope with stereopsis. It is unclear whether this revision method can maintain the bleb over the long term. It might be necessary to validate the use of adjuvant 5 -FU for better outcomes. These issues should be investigated in future studies.

\section{Acknowledgements}

We thank Prof. Maeda and Dr. Oshima for useful discussion. We thank Lynda Charters of Medical International for English language editing.

\section{Statement of Ethics}

The Ethics Committee of Osaka University Graduate School of Medicine approved the infrared CCD camera and monitor for clinical use and the study protocol. We got the patient's written informed consent to publish his case. 


\section{Case Reports in Ophthalmology}

\section{Disclosure Statement}

The authors have no conflicts of interest to declare in this report.

\section{Funding Sources}

The writing of the manuscript was supported in part by a Grant-in-Aid for Scientific Research (K.M.) and Global Center for Medical Engineering and Informatics in Osaka University (R.K.).

\section{Author Contributions}

Conception or design of the work: K.M.

Acquisition of data: K.M.

Analysis and interpretation of data for the work: R.K.

Drafting the work: K.M., R.K.

Revising it critically for important intellectual content: K.M.

Final approval of the version to be published: K.M., R.K., K.N.

\section{References}

1 Feldman RM, Tabet RR. Needle revision of filtering blebs. J Glaucoma. 2008 Oct-Nov;17(7):594-600.

2 Yamamoto T, Sawada A, Mayama C, Araie M, Ohkubo S, Sugiyama K, et al.; Collaborative Bleb-Related Infection Incidence and Treatment Study Group. The 5-year incidence of bleb-related infection and its risk factors after filtering surgeries with adjunctive mitomycin C: collaborative bleb-related infection incidence and treatment study 2. Ophthalmology. 2014 May;121(5):1001-6.

3 Hamanaka T, Omata T, Sekimoto S, Sugiyama T, Fujikoshi Y. Bleb analysis by using anterior segment optical coherence tomography in two different methods of trabeculectomy. Invest Ophthalmol Vis Sci. 2013 Oct;54(10):6536-41.

4 Shetty RK, Wartluft L, Moster MR. Slit-lamp needle revision of failed filtering blebs using high-dose mitomycin C. J Glaucoma. 2005 Feb;14(1):52-6.

5 Sagara T. An extra-fine crescent knife for bleb revision: bleb knife. J Ophthal Surg. 2010;23(1):71-4.

6 Ferrer H. Conjunctival dialysis in the treatment of glaucoma recurrent after sclerotomy. Am J Ophthalmol. 1941;24(7):788-90.

7 Broadway DC, Bloom PA, Bunce C, Thiagarajan M, Khaw PT. Needle revision of failing and failed trabeculectomy blebs with adjunctive 5-fluorouracil: survival analysis. Ophthalmology. 2004 Apr;111(4):665-73.

8 Watanabe-Kitamura F, Inoue T, Kojima S, Nakashima KI, Fukushima A, Tanihara H. Prospective 3D Investigation of Bleb Wall after Trabeculectomy Using Anterior-Segment OCT. J Ophthalmol. 2017;2017:8261364.

9 Shirakawa R, Arita R, Amano S. Meibomian gland morphology in Japanese infants, children, and adults observed using a mobile pen-shaped infrared meibography device. Am J Ophthalmol. 2013 Jun;155(6):1099-1103.e1.

10 Nomura H, Itoh N, Nomura N, Yasumura R, Takeda A, Endo Y, et al. Infrared ray imaging of scleral flaps after glaucoma surgeries. J Eye. 2011;28(6):879-82. 
Case Reports in Ophthalmology
Case Rep Ophthalmol 2020;11:234-241

(c) 2020 The Author(s). Published by S. Karger AG, Basel www.karger.com/cop

Matsushita et al.: Development of an Infrared Monitor-Guided Bleb Revision Procedure

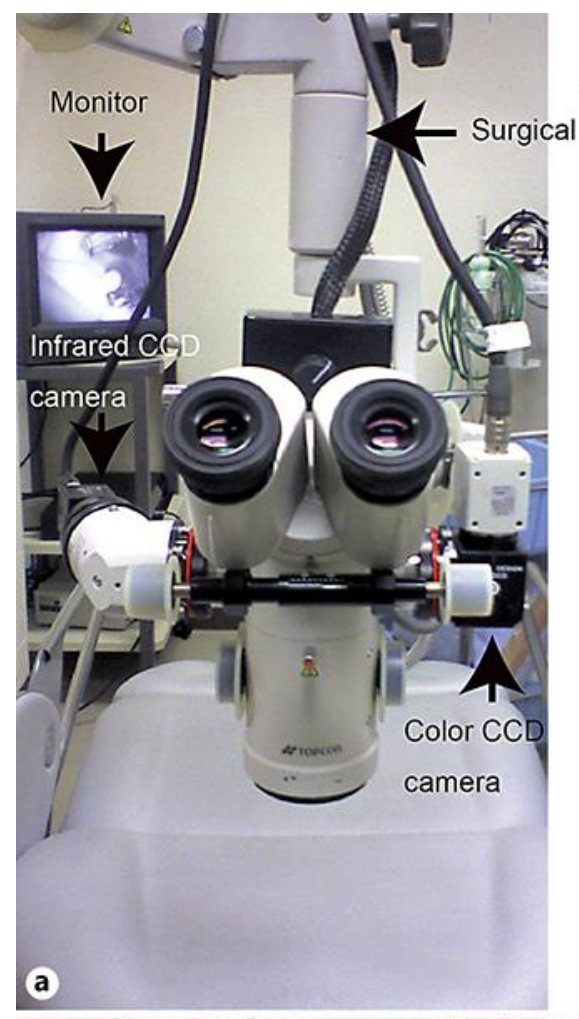

microscope
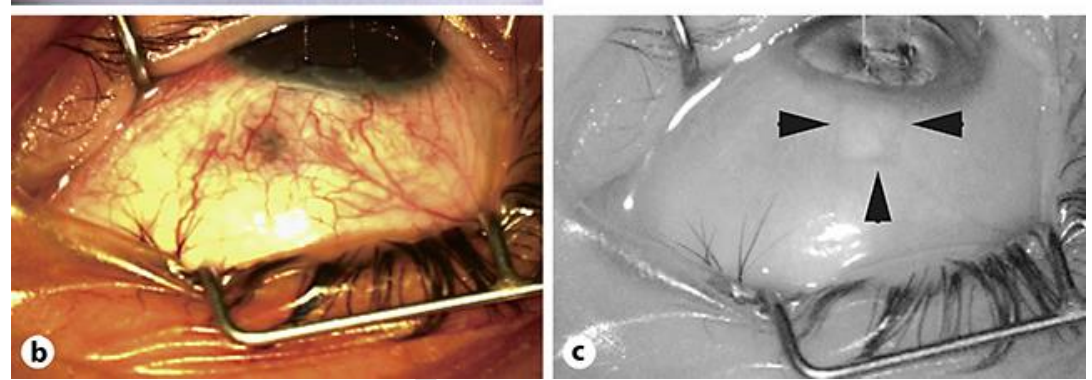

Fig. 1. The infrared CCD camera is embedded in a standard surgical microscope connected to the monitor (a). Comparison between visible light and infrared pictures. The monitor connected to the infrared CCD visualizes the site of the scleral filtering flap (arrowheads) (b, c). Visible light (b); infrared light (c). 
Case Reports in Ophthalmology
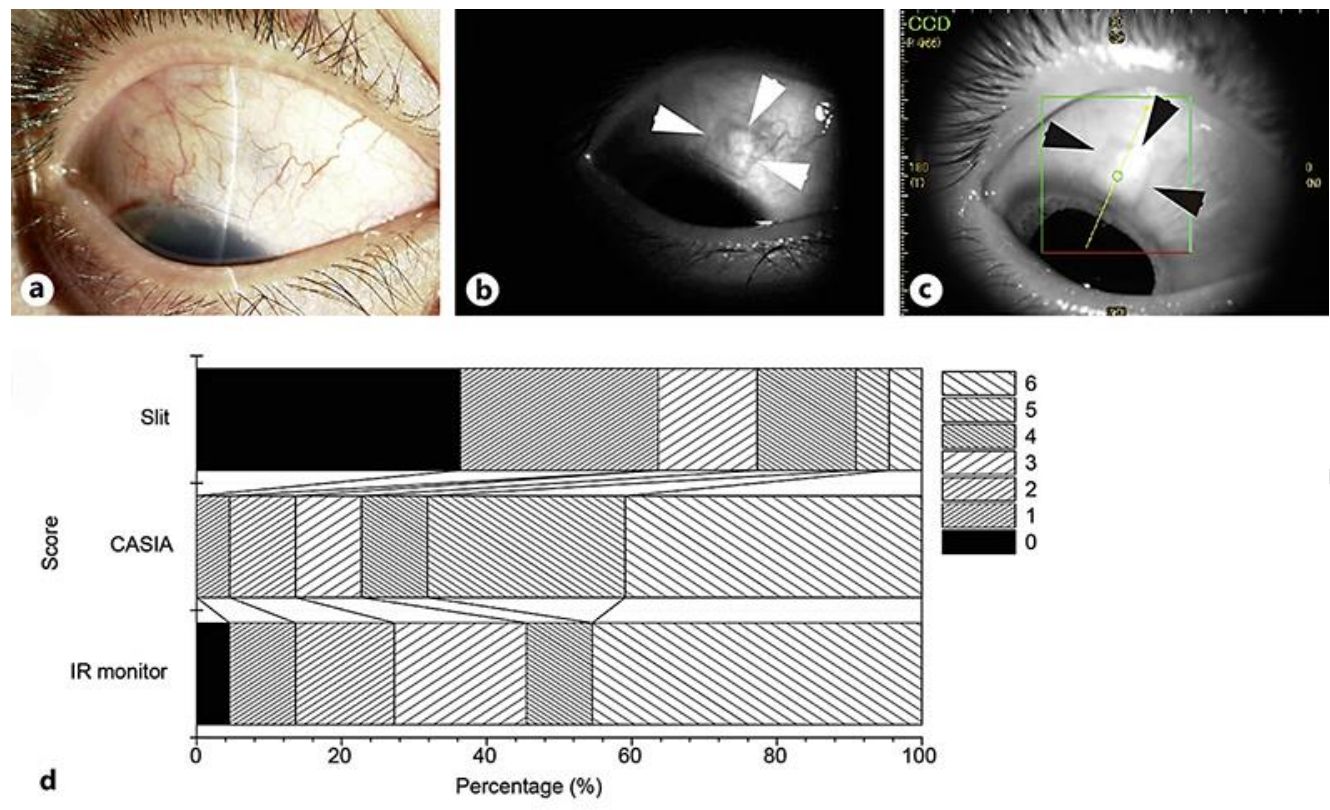

\begin{tabular}{|c|c|c|c|c|c|c|c|}
\hline Percentage score & 10 & 1 & 2 & 3 & 4 & 5 & 6 \\
\hline Slit & 36.4 & 27.3 & 0 & 13.6 & 13.6 & 458 & 4.5 \\
\hline CASIA & 0 & 4.5 & 9.1 & 9.1 & 9.1 & 27.3 & 40.9 \\
\hline IR monitor & 4.5 & 9.1 & 13.6 & 18.2 & 9.1 & 0 & 45.5 \\
\hline
\end{tabular}

\begin{tabular}{l|l|l|l} 
& Average score & $\begin{array}{l}\text { Percentage } \\
\text { over Score 1 (\%) }\end{array}$ \\
\hline Slit & 1.81 \\
\hline CASIA & 4.86 & $* *$ & 63.6 \\
\hline IR monitor & 4.05 & $* *$ & 100 \\
\hline
\end{tabular}

Fig. 2. The images under the infrared ray (IR) monitor and CASIA visualize the scleral flap (arrowheads). Slit (a). IR monitor (b). CASIA (c). The scores for flap visibility (d). The average scores and the percentages of the scores over $1(\mathbf{e}){ }^{* *} p<0.01$. 


\section{Case Reports in Ophthalmology}
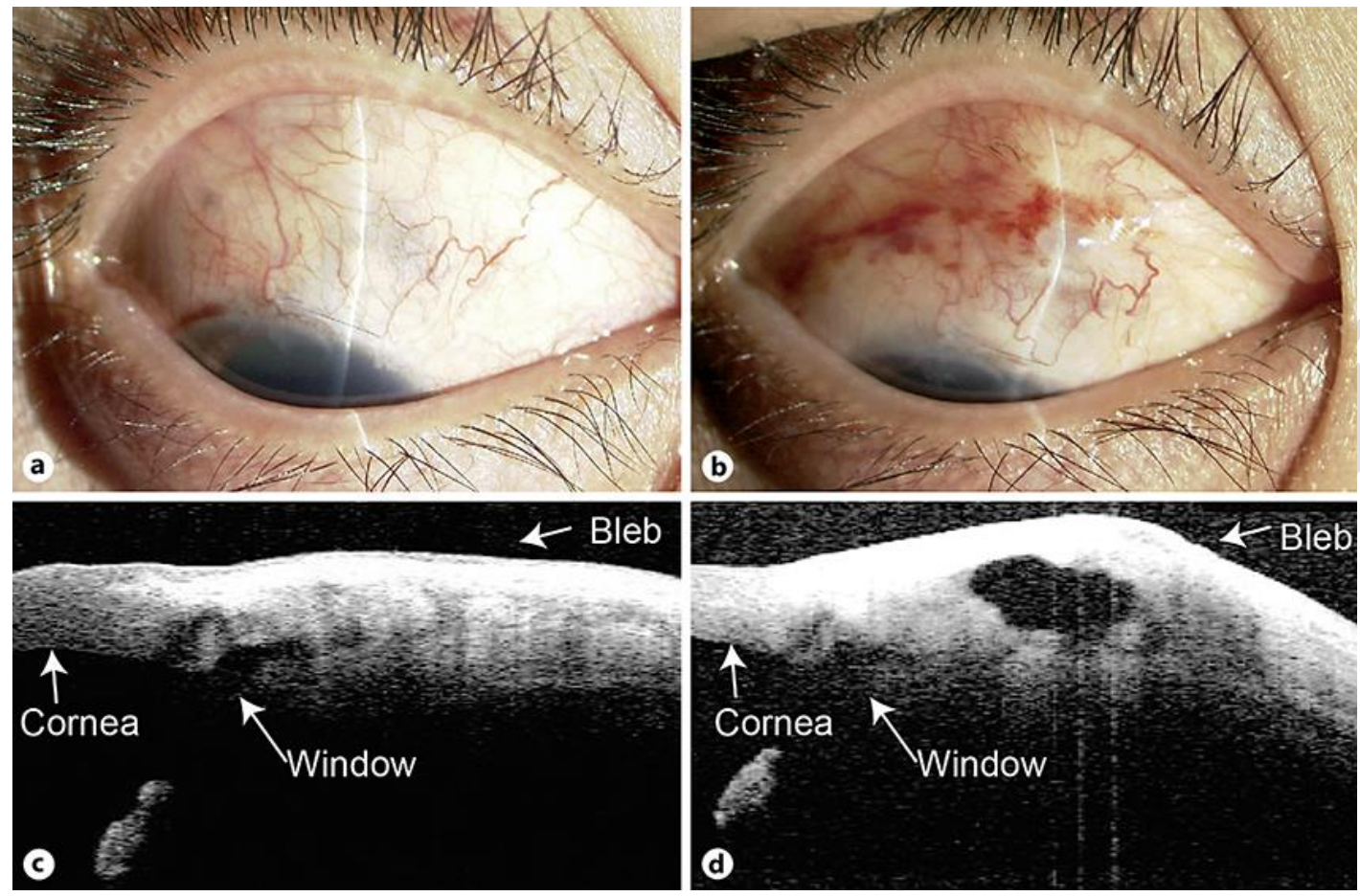

Fig. 3. Images from cases before and after the IGBR procedure. Slit-lamp photographs (a, b). Before needling (b). One month after needling (c). Bleb changes using the swept-source AS-OCT (SS-1000 CASIA). Before needling (c). One month after needling (d). 